\section{$\underset{\substack{\text { hommes } \\ \text { \& migrations }}}{ }$}

\section{Hommes \& migrations}

Revue française de référence sur les dynamiques

migratoires

$1313 \mid 2016$

1983, le tournant médiatique

\title{
Deux « Arabes » en vedette à la télévision
}

Karim Kacel et Smaïn

Yvan Gastaut

\section{Q OpenEdition \\ 12 Journals}

Édition électronique

URL : http://journals.openedition.org/hommesmigrations/3568

DOI : 10.4000/hommesmigrations.3568

ISSN : 2262-3353

Éditeur

Musée national de l'histoire de l'immigration

Édition imprimée

Date de publication : 1 janvier 2016

Pagination : 89-94

ISBN : 978-2-919040-34-6

ISSN : $1142-852 X$

Référence électronique

Yvan Gastaut, «Deux « Arabes » en vedette à la télévision », Hommes \& migrations [En ligne], 1313 |

2016, mis en ligne le 01 janvier 2019, consulté le 16 mars 2020. URL : http://journals.openedition.org/ hommesmigrations/3568; DOI : https://doi.org/10.4000/hommesmigrations.3568 


\title{
DEUX «ARABES 》 EN VEDETTE À LA TÉLÉVISION
} KARIM KACEL ET SMAÏN

Par YVAN GASTAUT, maître de conférences à l'URMIS, université de Nice, membre du comité de rédaction d'Hommes \& Migrations.

\author{
Au début des années 1980, alors que le débat sur l'immigration \\ mobilise l'opinion publique, la télévision française n'hésite \\ pas à mettre en lumière quelques jeunes issus \\ de l'immigration. Ainsi, de nouvelles figures apparaissent \\ dans nos petites lucarnes peu habituées à mettre en scène \\ des vedettes immigrées. Parmi elles, Karim Kacel et Smaïn \\ sont la preuve d'une réelle mutation du regard et des attentes \\ des téléspectateurs qui apprécient ces nouveaux talents \\ programmés à des heures de grande écoute.
}

Deux jeunes artistes, Karim Kacel et Smaïn, sauront s'illustrer et acquérir en peu de temps une notoriété inédite, qui dépasse largement le seul cadre du public « arabe » comme c'était le cas pour certains artistes auparavant ${ }^{1}$. Le succès s'offre à eux durant l'année 1983 : il sonne comme l'émergence de la part culturelle de l'immigration maghrébine à travers l'expression de la nouvelle génération née en France, porteuse d'une double identité. Dans le même esprit que l'exposition des artistes issus de l'immigration organisée la même année au Centre Georges-Pompidou et concomitante de la Marche contre le racisme et pour l'égalité, cette éclosion est le signe de l'acceptation d'une France plurielle par une partie de l'opinion ${ }^{2}$.

\section{Karim Kacel, poète de la banlieue}

Karim Kacel, né en 1959 dans le XIVe arrondissement de Paris, est l'aîné d'une famille algérienne installée en France durant les Trente Glorieuses. Son père est un ouvrier kabyle analphabète et sa mère originaire d’Alger. Ayant grandi au Kremlin-Bicêtre, il quitte l'école à lâge de 16 ans et devient autodidacte, il lit beaucoup tout en pratiquant la boxe. Son souhait est de devenir éducateur. Dans le même temps, la musique le passionne : il apprend la guitare en solitaire, écrit des textes et des poèmes et 
s'essaie à la chanson. Le jeune homme fait d'abord chanter les enfants puis prend progressivement confiance en ses capacités. En 1982, à lâge de 23 ans, Karim Kacel décide de s'inscrire à un télécrochet organisé par l'émission d’Antenne 2 Moi, je..., magazine de société produit par Pascale Breugnot et composé de petits documentaires sur " l'air du temps ». Le concours tourne en faveur de Karim Kacel : séduits, les téléspectateurs votent massivement pour lui et le classent premier. Tout auréolé de ce succès, il est sollicité par un directeur artistique de la maison de disques Pathé Marconi qui lui propose d'enregistrer un 45 tours. Ce sera Banlieue, qui va connaître un immense succès populaire dès le début de 1983. Kacel offre un regard de l'intérieur, celui d'un jeune qui éprouve son cadre de vie : "Il regarde sa ville / Tranquille / Et il attend / Il sait qu'il est fragile, difficile / Et pourtant / Il ouvre ses grands yeux / Et regarde sa banlieue / Le chômage à son âge / Ne le rend pas heureux / Cet horizon de tours, qui l'entoure / L'asphyxie / Son univers est lourd, passent les jours / Et l'ennui / Ce n'est qu'un enfant, qui rêve de grands vents / Donnez-lui de l'espace, qu'il efface ses tourments. " Cette chanson est emblématique du début des années 1980 marqué par la question des grands ensembles et des premières émeutes urbaines datant de 1981, notamment dans la banlieue lyonnaise. Face aux inquiétudes sur les dérives délinquantes des jeunes issus de l'immigration, le public adhère et apprécie cette chanson émouvante, la voix chaleureuse de son interprète au visage doux. La sensibilité et la sincérité du propos n'échappent à personne : "De café en café, avec des paumés / Il passe son temps / Il se saoule un p’tit peu, joue avec le feu / Joue au délinquant / C'est pas qu’il soit méchant / Demandez aux parents / Mettez-vous à sa place / C'est dur de faire face / Quand on a qu'dixsept ans / Le vol des mobylettes, on fait la fête / Sur le moment / La police le guette, ses parents s'inquiètent / Comme dans un roman / Regarde, c'est ton enfant / C'est le sang de ton sang / C'est toi qui l'as nourri et jeté dans la vie / Il n'y a pas si longtemps / Hé banlieue, ne nous laisse pas vieillir / On a peur de mourir, banlieue / Hé banlieue, ta grisaille nous inspire / Que l'envie de partir, banlieue / Hé banlieue, ne nous laisse pas tomber / On a l'droit d'exister nous aussi / Banlieue... ohhh... banlieue. »Ce cri quelque peu mélancolique d'un "jeune immigré " trouve une bonne place dans la bande-son de cette époque.

Comme il le raconte dans une autre édition de Moi, je..., le 19 janvier 1983, intitulée « Coup de chance pour Karim ", l'auteur ne fait qu'évoquer sa vie quotidienne. Il le confesse : Banlieue, c'est lui! Les vols de mobylettes, la galère, c'est bien son histoire personnelle qui est mise en scène. Et s'il s'en est sorti, c'est grâce aux amitiés qu'il a nouées dans cet univers plutôt sombre. Dans cette émission, d'emblée, la question cruciale lui est posée : "Vous vous sentez plutôt quoi, français ou algérien? " En guise de réponse, un grand embarras: Kacel affirme avec son accent parisien qu'il est algérien avant tout, que ses racines sont de l'autre côté de la Méditerranée, mais que sa vie est en France, sa culture est française, qu'il compose ses chansons en français et non pas dans la langue arabe, que d'ailleurs il ne connaît pas. Pourtant, si l'on retourne la pochette du disque réalisée par le photographe Jean Eckian, la face B, moins connue, est tout aussi importante pour saisir le personnage. On y découvre La chanson du Kabyle, un hommage sensible rendu à son père "descendu des montagnes » à lâge de 12 ans pour venir travailler en France, " on l'appelait l'immigré ». L'autre part de son expérience de la double culture : à travers cette seconde face, son ambition est de préserver sa mémoire, afin que ses enfants et petits-enfants n'oublient pas que ce père " a été le premier », selon les propos tenus par l'artiste dans Moi, je...

\section{Chanter la double identité}

À l'heure de l'émergence de la France multiculturelle, c'est sur le thème de cette double appartenance qu'il faut valoriser Karim Kacel, les journalistes en sont alors convaincus. "Parce qu'il chante la banlieue de sa jeunesse et chante 
aussi son pays natal, la Kabylie de ses ancêtres, et qu'il n'est pas facile de chanter français quand on est d'origine algérienne ", affirme maladroitement la présentatrice Marie-Laure Augry pour présenter Karim Kacel, qui fait l'objet d'un reportage "Coup de cœur » au Journal télévisé de 13 heures de TF1, le 6 juillet 1983. La rédaction et le service culture de TF1 ont, en effet, repéré dans le chanteur "beur » une figure positive de l'intégration. Karim Kacel apparait comme le «bon client » arabe des médias : poète, calme et intégré.

Voilà une chanson qui apporte un témoignage positif sur ces « jeunes Beurs » qui inquiètent une partie des Français quant à leur capacité d'intégration. Après un passage à Aujourd'hui la vie le 2 mai 1983 en début d’après-midi sur Antenne 2, où il s'entretient avec Dominique Verdeilhan avant d'interpréter Banlieue, puis, à nouveau, le 7 novembre, dans une émission spéciale sur les Algériens en France à l'occasion de la visite officielle à Paris du président Chadli Benjedid, Karim Kacel se retrouve sur France 3 dans La Vie à plein temps, le 5 mai. Dans cette nouvelle émission de grande écoute, il est aux côtés de Mehdi Charef, qui vient de publier son premier roman, Le Thé au harem d'Archi Ahmed', ayant lui aussi obtenu un important succès. Appartenant à la même génération, ayant vécu des parcours similaires marqués par les grands ensembles, la discrimination, mais aussi par l'école et les amitiés adolescentes, les deux hommes décident de collaborer lorsque le roman de Medhi Charef devient un film, Le Thé au harem d'Archimède. Sortie dans les salles en 1985, cette fresque sociale décrit la vie quotidienne d'une cité HLM de jeunes de banlieue, qu'ils soient français ou d'origine immigrée. Madjid, fils d'immigrés et aîné d'une famille nombreuse entièrement soutenue par la mère et son meilleur ami Pat, essaie de se dessiner un avenir. Malgré quelques mauvais penchants et le risque de sombrer dans la délinquance dans un contexte de désarroi généralisé, le film insiste sur les amitiés solides entre les jeunes et une certaine humanité se dégage de ces pérégrinations. Logiquement, la chanson Banlieue devient la bande-son d'un film qui sera un très grand succès. Après l'avoir entendue pendant toute l'année 1983, les Français la retrouvent dans le film de Medhi Charef en 1984.

Karim Kacel passe par tous les lieux qui contribuent à faire de lui un chanteur populaire, notamment le 28 avril 1984 dans lémission la plus populaire du moment, Champs-Élysées, présentée par Michel Drucker, après avoir participé au printemps de Bourges 84 et avant d'être à l'affiche au Théâtre de la Ville et d'effectuer entre 1984 et 1986 de nombreuses tournées en France mais aussi à l'étranger.

Ses nouvelles chansons tentent par la suite de s'inscrire dans le répertoire classique de la chanson française,
Malgré quelques mauvais penchants et le risque de sombrer dans la délinquance dans un contexte de désarroi généralisé, le film insiste sur les amitiés solides entre les jeunes et une certaine humanité se dégage de ces pérégrinations. davantage portés vers une musicalité et des sons aux tonalités orientales comme le groupe Carte de séjour. En 1986, Karim Kacel reçoit successivement le prix Charles Cros pour sa chanson P'tite sour tirée de son deuxième album 33 tours et le prix Georges Brassens. Ce succès lui vaut un nouveau passage à Champs Élysées, le 29 mars 1986. Karim Kacel retrouve le printemps de Bourges entre 1986 et 1988 : comble d'honneur, le 20 avril 1987, le président Mitterrand, en visite privée surprise depuis Latche, assiste au concert ${ }^{4}$. Le président, qui tient à le rencontrer à l'issue du concert, se déclare devant les caméras "séduit et emporté par le rythme de Kacel ». Lorsqu'il se produit à l'Olympia en 1988, il obtient le prix Édith Piaf du meilleur spectacle de l'année puis, en 1989, il reçoit des mains de Léo Ferré le prix du meilleur parolier décerné par le Petit Robert.

Cependant, à partir de la fin des années 1980, la mode « beur » s'étant quelque peu estompée, la carrière de Karim Kacel ne connaîtra plus autant d'éclat auprès du grand public. Si le chanteur 
poursuit sa carrière, sa notoriété marque le pas puis décline : ses passages en prime time se raréfient malgré la sortie de 8 albums entre 1991 (Ruses de Sioux) et 2008 (Bluesville). En 2015, il sort un nouvel album intitulé Encore un jour. On le voit, Karim Kacel n’a pas renoncé à sa carrière comme le montre un ouvrage du jourSmaïn apparaît naliste Gilbert Jouin qui retrace pour la première fois

à l'écran le 26 février 1983

sous le parrainage de

Darry Cowl.

Il interprète le rôle

d'un balayeur arabe,

Monsieur Ali. son parcours en $2008^{5}$, année au cours de laquelle il interprète le répertoire d'un autre chanteur issu de l'immigration, Serge Reggiani.

Pour le grand public, Karim Kacel restera le chanteur d'un tube, emblématique de l'émergence des jeunes issus de l'immigration, à l'instar de la chanson de Renaud Deuxième génération sortie au même moment en 1983 : "J'm'appelle Slimane et j'ai 15 ans, j'vis chez mes vieux à La Courneuve... ", qui illustre également les tourments de l'entre-deux identaire : "Des fois j' me dis qu'à trois mille bornes / De ma cité y a un pays / Quej'connaîtrai sûrement jamais / Que p't-être c'est mieux, que p't-être c'est tant pis / Qu'là-bas aussi j'serai étranger / Qu'làbas non plus je serai personne /Alors pour m' sentir appartenir / A un peuple à une patrie / J' porte autour d' mon cou, sur mon cuir / le keffieh noir et blanc et gris / J' me suis inventé des frangins / Des amis qui crèvent aussi. »

\section{Smaïn ou l'incarnation comique des travailleurs immigrés}

L’année de la révélation de Karim Kacel, 1983 est également marquée par la popularité de Smaïn dans Le Petit Théâtre de Bouvard. Programmée chaque soir de la semaine sur Antenne 2 à 19 h 45 - en access prime time - entre septembre 1982 et juin 1985, cette émission réalisée par Nino Monti se donne pour objectif d'attirer le maximum de téléspectateurs devant le journal télévisé

de 20 heures de la chaîne. Envisagée au départ autour de discussions de comptoir entre Philippe Bouvard et ses invités, l'émission va finalement se fonder sur une succession de sketches loufoques pendant une quinzaine de minutes. Devant un public conquis, ces scénettes, parodies ou pastiches, souvent improvisées, sont interprétées par une multitude de jeunes comédiens prometteurs encadrés par un illustre invité. Ne manquant pas de tourner en dérision l'air du temps, Le Petit Théâtre de Bouvard trouve son public et connaît un rapide et énorme succès d'audience. En outre, le talent de cette troupe de comédiens alors peu connus contribue au succès de l'émission. Recrutés sur audition après avoir été repérés sur les différentes scènes des cabarets parisiens, ils sont les représentants d'une nouvelle génération d'humoristes devenus populaires : Muriel Robin, Mimi Mathy, Michèle Bernier, Philippe Chevalier, Régis Laspallès, Pascal Légitimus, Didier Bourdon, Bernard Campan, Bruno Gaccio, Jean-Marie Bigard ou encore Laurent Gamelon, pour les plus connus. Smaïn apparaît pour la première fois à l'écran le 26 février 1983 sous le parrainage de Darry Cowl. Il interprète le rôle d'un balayeur arabe, Monsieur Ali - «Ali comment? Ali la France»-, exalté par sa profession au point d'avoir crée un "corps de balais". Singeant de manière outrancière l'accent arabe, son apparition s'avère convaincante. Elle se situe dans le sillage du succès des sketches de Pierre Péchin, que ce soit S'il vô plaît!, lorsqu'il incarne un travailleur immigré balayant les rues en 1974 ,ou son adaptation de La cigale et la fourmi avec l'accent arabe à partir de 1975, qui connut un immense succès. Imiter les travailleurs immigrés maghrébins continue à faire rire en ce début des années 1980 et le jeune Smaïn, à la différence de Pierre Péchin, décide de s'y employer en jouant sur son physique, ce qui plaît au public.

Smaïn devient un comédien régulier de cette émission durant toute l'année 1983 et se révèle ainsi au grand public. Avec Pascal Légitimus (né à Paris d'un père martiniquais et d'une mère d'origine 
arménienne) et Tchee (de son nom complet Tchee Meas), d'origine cambodgienne, ce jeune acteur " issu de l'immigration » âgé de 25 ans symbolise une certaine forme de diversité à la télévision, reflet de la France plurielle émergeante. Le 4 mars, il interprète un jeune « Beur » victime du racisme des policiers ; le 25 mars, un jeune des cités qui cherche la bagarre sur un terrain vague, ou encore, le 4 juillet, un Dom Juan dans une boîte de nuit de banlieue. Le $1^{\text {er }}$ juin, il interprète un Arabe qui cherche sa route et demande la "rue Montalembert ou rue monte à l'envers ». Ainsi, indistinctement, Smaïn joue à « l'Arabe de service ", qu'il soit le travailleur immigré de la première génération ou, plus crédible, le "Beur ».

Mais ne nous y trompons pas : à regarder l'ensemble des prestations de l'humoriste, celui-ci n'interprète pas seulement des rôles d'Arabe. Il apparaît également sous les traits d'un banquier, monsieur Lemercier, qui rétablit "l'équilibre monétaire " (en faisant tenir un billet sur la tranche en équilibre sur son front), d'un enseignant, d'un amoureux transi, d'un dragueur invétéré, d'un homosexuel, d'un patient chez un spécialiste de chirurgie esthétique ou encore de d'Artagnan et du prince charmant dans Blanche-neige et les sept nains, dans des situations non ethnicisées. Le 19 novembre, il imite Yves Montand en parodiant ses chansons sous le regard bienveillant de Bernard Haller. Dans les compositions, Smain fait souvent équipe avec les futurs «Inconnus » (Didier Bourdon, Pascal Légitimus, Bernard Campan et Serge Brussels), avec lesquels il monte même un groupe à l'existence éphémère, "Les Cinq ». Après une année d'omniprésence dans les petites lucarnes à l'heure du dîner, Smaïn est devenu célèbre. Le Petit Théâtre de Bouvard a été pour lui un véritable tremplin médiatique. Le contexte lui est favorable. Smain s'apparente ainsi, pour le grand public, à l'un de ces jeunes issus de l'immigration qui réussit grâce à son talent. Ne voulant pas s'endormir sur ses lauriers, l'acteur décide dès le début de l’année 1984 de poursuivre sa carrière d'une autre manière, en solo, profitant de cette notoriété soudaine.

\section{De l'orphelinat aux feux de la rampe}

Mais qui est cet «Arabe » qui « crève l'écran » en 1983 ? Smaïn Faïrouze est né en 1958 à Constantine de père et de mère inconnus, comme il le raconte dans son autobiographie publiée en 2011, Je reviens me chercher ${ }^{6}$. Après avoir été recueilli dans un orphelinat catholique de Constantine, le jeune Algérien arrive en France à lâge de 2 ans en 1960, où il est adopté par un couple sans enfants, un balayeur algérien employé à Paris et son épouse marocaine femme de ménage : "D’origine modeste, ils regardaient avec méfiance le monde des artistes. Ils me répétaient sans cesse qu'il y a beaucoup d'appelés et peu d'élus... Mais mon désir a vite eu raison de leurs conseils! Malheureusement ils sont morts trop tôt pour pouvoir profiter de ma réussite. Mais jespère qu'ils en auraient été fiers ${ }^{7}$. " Placé à l'école NotreDame puis au lycée Saint-Michel de Saint-Mandé, le jeune Smaïn Faïrouze chante au sein de la chorale des Petits Chanteurs de la renaissance avant de se passionner pendant son adolescence pour les arts du spectacle.

Ce n'est pas un hasard si sa carrière commence dans le café-théâtre : il

se produit à partir de lâge de 22 ans dans de modestes cabarets parisiens. On lui ferme les portes du Conservatoire mais, au cabaret, on lui demande, pour faire rire, de prendre l'accent arabe même s'il ne parle pas couramment cette langue. Comme il le précise dans son autobiographie : «Il fallait que je joue sur ma spécificité8. " En 1982, il décroche un rôle secondaire dans le film de Francis Girod Le Grand Frère, avec Gérard Depardieu et Souad Amidou : Smaïn interprète le personnage d'Ahmed dans le Marseille multiculturel du début des années 1980 où se pose la question du racisme 
envers les jeunes issus de l'immigration. Plus tard, Smain jouera d'autres rôles d' "Arabe " au cinéma comme dans La Smala de Jean-Loup Hubert en 1984, Le téléphone sonne toujours deux fois! de JeanPierre Vergne en 1985, Les Frères Pétard d'Hervé Palud en 1986, On peut toujours rêver de Pierre Richard en 1990 et, surtout, L'CEil au beur(re) noir de Serge Meynard en 1987, qui reçoit le César du meilleur premier film l'année suivante. Dans cette comédie, il joue le rôle principal, celui d’un « Beur » subissant diverses formes de discriminations et qui peine à vivre au quotidien à Paris en enchaînant petits boulots et déconvenues amoureuses.

À côté de son succès au cinéma, Smaïn excelle au même moment dans le one man show. En 1986 notamment, sa première expérience en la matière, le spectacle A Star is Beur attire un large public sur la scène du café-théâtre le Tintamarre à Paris. Plusieurs sketches tirés de ce spectacle sont diffusés à la télévision comme, par exemple, dans Aujourd'hui la vie le 10 octobre 1986 ou dans Champs-Élysées le $1^{\text {er }}$ novembre ${ }^{9}$. La mise en scène de l'élocution quelque peu maladroite d'un président de la République arabe ou d'un "président beur » au fort accent arabe suscite, en particulier, l'hilarité : « Chers Françaises, chers François, vous m'avez élu au chauffage universel, je tiens à vous dire merci. " Dans les années 1990, bien ancré dans le monde du théâtre, du caféthéâtre et du cinéma, Smaïn reste sur le devant de la scène, souvent invité sur les plateaux de télévision dans des émissions grand public. Que ce soit au Petit Théâtre de Bouvard, au cinéma ou sur scène, le fil rouge de l'humour de Smaïn consiste à détourner les stéréotypes en les marquant outrageusement. Il s'agit d'un humour de l'entre-deux : lorsqu'il joue à l'Arabe, c'est pour mieux accentuer les préjugés afin de les détourner ; lorsqu'il joue des rôle non ethnicisés c'est pour mieux prouver qu'un «Arabe » peut incarner n'importe qui. Fort de cette position, en 1992, il obtient un Molière et un trophée aux Victoires de la musique, tandis qu'en 1994-1995 il joue le rôle principal dans Les Fourberies de Scapin, dans une mise en scène de Jean-Luc Moreau au théâtre du Gymnase puis en tournée dans toute la France. Néanmoins, sa notoriété commence à décliner à partir du début des années 2000. S'il n'a pas disparu du paysage audiovisuel et culturel français, sa présence se fait plus rare. Quelques films (Rebelote en 2004 et Mon dernier... avant le prochain en 2011), quelques pièces de théâtre qu'il met en scène luimême (Réactions en chaîne en 2011-2012 au Théâtre Rive Gauche et en tournée, et La Ménagère apprivoisée en 2013 au théâtre l'Archipel avec Jean-Marc Longval et Éric Carrière) ne suffisent pas à maintenir le succès de Smaïn au niveau qui était le sien dans les années 1980.

\section{Conclusion}

Le succès de Karim Kacel et de Smaïn apparu en 1983 et développé les années suivantes s'inscrit dans le contexte de l'émergence d'une culture populaire qui prend en compte l'apport de l'immigration par le biais de jeunes artistes témoignant par leur talent de leur condition de Français d'origine immigrée. Les aspirations multiculturelles d'une partie de l'opinion sont bel et bien relayées par les médias, en particulier par la télévision : c'est la brève période du "Beur is beautiful », que l'on peut situer entre 1983 et 1989, faisant des jeunes issus de l'immigration les tenants d'un nouveau souffle créatif en France dans tous les domaines. À l'issue de cette période, à l'instar d'autres figures de ce «mouvement beur », la notoriété de Karim Kacel et, à un moindre degré, celle de Smaïn tendent à s'estomper : leurs carrières se poursuivent de manière plus confidentielle. Les artistes de cette génération «beur » peinent à conserver leur notoriété : est-ce le signe d'un essoufflement de leur créativité qui n'est plus en phase avec le public populaire des décennies suivantes ou celui d'une parenthèse refermée sur ces questions ? Quoi qu'il en soit, la grande popularité de ces deux artistes en 1983 informe l'historien sur le rapport de la société française à l'immigration au cours de ces "années tournantes ». 